\title{
The effect of a low dielectric material placed at the tip of a GTEM cell on the electric field
}

\author{
Ifong $\mathbf{W u}^{\mathrm{a})}$, Shinobu Ishigami, Kaoru Gotoh, \\ and Yasushi Matsumoto \\ EMC Group, National Institute of Information and Communications Technology \\ 4-2-1 Nukui-Kitamachi, Koganei, Tokyo 184-8795, Japan \\ a) ifong@nict.go.jp
}

\begin{abstract}
The effect of a low dielectric block placed at the tip of a GTEM cell on the electric field was numerically and experimentally investigated from 1-16 GHz. The block increased the electric field strength when placed at the GTEM cell tip. Comparison of the simulation and measurement results revealed good agreement between them. However, we also confirmed that when the low dielectric block was installed in a location far from the GTEM cell tip, it had little influence on the electric field, regardless of its size. It is concluded that a low dielectric material increases the electric field strength when it is placed at the tip of a GTEM cell.
\end{abstract}

Keywords: GTEM cell, low dielectric block, permittivity, electric field

Classification: Electromagnetic compatibility (EMC)

\section{References}

[1] T. Loh and M. J. Alexander, "A method to minimize emission measurement uncertainty of electrically large EUTs in GTEM cells and FARs above $1 \mathrm{GHz}$," IEEE Trans. Electromagn. Compat., vol. 48, pp. 634-640, Nov. 2006.

[2] H. Kamiya, M. Yamada, M. Tokuda, S. Ishigami, K. Gotoh, and Y. Matsumoto, "Evaluation of interference between MB-OFDM UWB and wireless LAN systems using a GTEM cell," 2008 IEEE Int. Electromagn. Compat. Symp., THU-PM-2-5, Detroit, 18-22 Aug. 2008.

[3] IEC 61000-4-20, Electromagnetic compatibility (EMC)-Part 4-20: Testing and measurement techniques-Emission and immunity testing in transverse electromagnetic (TEM) waveguide, 2003.

[4] IEEE Standards 1309: IEEE standard for calibration of electromagnetic field sensors and probes, excluding antennas, from $9 \mathrm{kHz}$ to $40 \mathrm{GHz}, 2005$.

[5] Elena Electronics Co., LTD. website. [Online] http://www.elena-e.co.jp/ 3posi.pdf/

[6] X.T.I. Ngu, A. Nothofer, D.W.P. Thomas, and C. Christopoulos, "A complete model for simulating magnitude and phase of emissions from a DUT placed inside GTEM cell," IEEE Trans. Electromagn. Compat., vol. 49, 


\section{Introduction}

With the innovative miniaturization and high-speed operation of recent electrical devices, the clock speeds of computer processors have been extended beyond $1 \mathrm{GHz}$ [1] , and the ultra-wideband (UWB) devices are up to $10.6 \mathrm{GHz}$ [2]. Hence, the leakage of electromagnetic field radiation from these devices has also entered the high frequency range and often leads to serious electromagnetic interference (EMI) problems with other wireless devices. Therefore, it is necessary to measure emissions and perform immunity testing up to several $\mathrm{GHz}$ for these devices. A gigahertz transverse electromagnetic (GTEM) cell has recently been used as alternative apparatus to an open area test site (OATS) and a semi-anechoic chamber (SAC) for measuring the radiated emission or immunity testing [3]. Compared with conventional transverse electromagnetic (TEM) waveguides, there are no major geometrical discontinuities and higher order mode excitations are not evident in GTEM voltage standing wave ratio (VSWR) measurements below $20 \mathrm{GHz}$ [4]. Therefore, it is possible to use a GTEM cell to measure emissions and perform immunity testing for frequencies from DC to several GHz. To measure emissions and perform immunity testing, the electric field uniformity is necessary in a GTEM cell. In order to maintain the electric field uniformity in a GTEM cell, the stable form of the septum is required. Generally, the septum is suspended from the ceiling with thread and a low dielectric block such as a foam block is placed at the tip of the GTEM cell to maintain the shape of the septum. Furthermore, the low dielectric material is also used to adjust the electric field distribution by placing it at the tip of the GTEM cell. There is no clear effect on the electric field from the low dielectric material at low frequencies [5]. However, when the measurement frequency increases, the electric field may be affected by the low dielectric material. There have been very few studies on the effect of a low dielectric material on the electric field in a GTEM cell. In this study, we numerically and experimentally investigated the effect of a low dielectric block placed at the tip of a GTEM cell on the electric field in the $1-16 \mathrm{GHz}$ frequency range. First, the effect of various permittivity values for the low dielectric block on the electric field was conducted by the simulation. Using the simulation is more convenience to evaluate and easier to calculate the effect of permittivity values for various frequencies at one time than measurement. In order to save the time and reduce the computational resources, this simulation modeled only the tip of the GTEM cell. We then demonstrated that the effect of a low dielectric block on the electric field was accurately evaluated by simulation by comparing with the measurement. We also experimentally evaluated the effect when the block is placing far from the tip of the GTEM cell. 


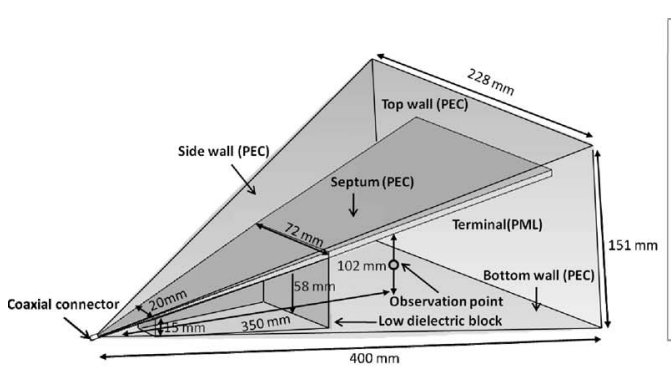

(a) Tip of the GTEM with a low dielectric block

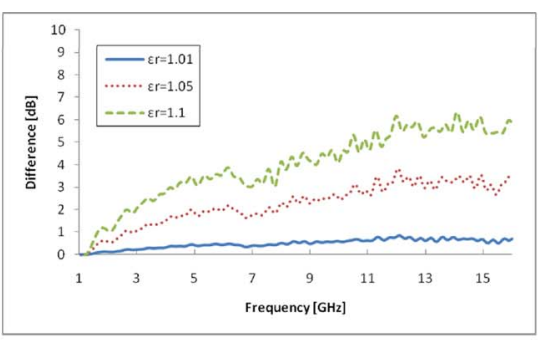

(b) Effect on the electric field of various electric permittivity values

Fig. 1. Model construction and simulation result.

\section{Simulation of effect on electric field of low dielectric block}

In this section, the effect of a low dielectric block placed at the tip of a GTEM cell on the electric field was numerically investigated. To reduce the computational resources required for the numerical analysis, we modeled only the tip of the GTEM cell and used this model to investigate the change of the electric field in the GTEM cell.

\subsection{Simulation model}

Fig. 1 (a) shows schematic of the tip of a GTEM cell with a low dielectric block. This model has a coaxial connector, two side walls, top wall, bottom wall, and a septum. The simulated impedance of the GTEM cell was $50 \Omega$. The metallic walls and the septum were assumed to be a perfect electric conductor (PEC). To avoid reflected waves from the end of the GTEM cell, a perfectly matched layer (PML) with four layers was used in the model [6]. The low dielectric block was assumed to be a homogeneous medium. The block was set $50 \mathrm{~mm}$ from the coaxial connector and filled up the space between the bottom wall and the septum. In addition, the nonuniform grid method was applied in this calculation. The simulation was carried out from 1-16 GHz. A constant net power of $1 \mathrm{~W}$ was supplied from the coaxial connector. Furthermore, the observation point was set in the middle, where the distance between the bottom wall and the septum was $102 \mathrm{~mm}$.

\subsection{Calculation of the effect on the electric field}

To calculate the effect of the low dielectric block on the electric field, the finite integration (FI) method was applied. Fig. 1 (a) shows the simulation model used for calculating the effect on the electric field at the observation point. The observation point was $350 \mathrm{~mm}$ from the coaxial connector and centered between the bottom wall and the septum, which were $102 \mathrm{~mm}$ apart. To evaluate the effect on the electric field, the change of the electric field at the observation point was defined as follows:

$$
E_{\text {diff }}[d B]=E_{\text {withblock }}-E_{\text {withoutblock }}
$$

where $\mathrm{E}_{\text {diff }}$ is the amount of change in the electric field from the low dielectric block, $\mathrm{E}_{\text {withblock }}$ is the electric field strength at the observation point with the block (Fig. 1(a)), and $\mathrm{E}_{\text {withoutblock }}$ is the electric field strength at the 
observation point without the block.

\subsection{Effect of various permittivity values for the low dielectric block on the electric field}

Generally, the low dielectric material like polystyrene foam block will not affect the propagation of the electromagnetic wave at low frequency. When the frequency increases, the low dielectric block may affect the electric field by placing it at the tip of GTEM cell. In this section, we discuss various permittivity values for the block and examine their effect on the electric field in the $1-16 \mathrm{GHz}$ frequency range. We assume the permittivity values $\left(\varepsilon_{\mathrm{r}}\right)$ of the low dielectric block to be 1.01, 1.05, and 1.1. In order to compare the electric field, the net power is controlled to be $1 \mathrm{~W}$. Fig. 1 (b) shows the result of the effect of various permittivity values on the electric field. We found that the electric field strength increased when the block was placed at the tip of the GTEM cell. We also found that the electric field strength increased as the permittivity value increased. Furthermore, the electric field strength increased with an increase in frequency. This increase may have been caused by multiple reflections between the coaxial connector and the tip of the block. When the frequency increased, the effect on the electric field also increased.

\section{Measurement of effect on electric field}

The low dielectric block increased the electric field, as described in section 2. This section discusses an investigation to determine the effect on the electric field by measurements. We then compare the results obtained from the simulation and measurement.

\subsection{Measurement procedures}

Fig. 2 (a) shows a schematic diagram of the measuring system. A signal generator was connected to a power amplifier to amplify the input signal. The power amplifier was connected to a dual directional coupler to calculate the input signal and the dual directional coupler was connected to the tip of the GTEM cell to input the signal. The net power $\left(\mathrm{P}_{\text {net }}\right)$ is defined as follows:

$$
P_{n e t}[W]=P_{f w d}-P_{r e v}
$$

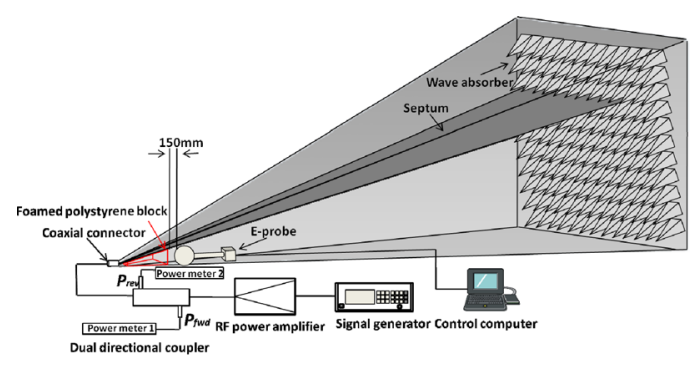

(a) Measurement system

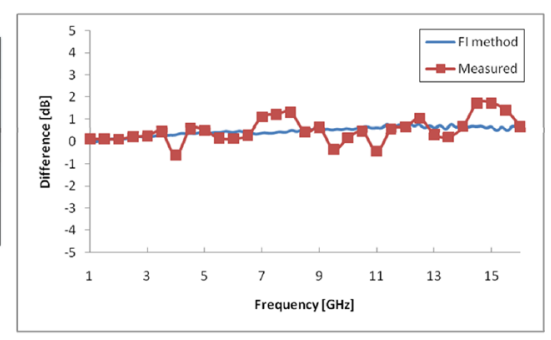

(b) Comparison result

Fig. 2. Measurement system and comparison result. 
where $\mathrm{P}_{\text {net }}$ is calculated from the forward power $\left(\mathrm{P}_{\mathrm{fwd}}\right)$ and the reverse power $\left(\mathrm{P}_{\text {rev }}\right)$, which are measured by the power meter 1 and 2 . In this study, the net power is set at $1 \mathrm{~W}$. The block was placed in the same position as in the simulation model, as shown in Fig. 1 (a), and the structure of the block was also the same as in Fig. 1(a). We assumed the commercially supplied polystyrene block to be a low dielectric block $\left(\varepsilon_{\mathrm{r}}=1.01\right)$ and used it in the measurements. To avoid the effect of the coupling between the probe and metal walls, the measurement point is set at a point centered between the bottom wall and the septum as an example. An electric field probe was set in the GTEM cell in a plane where the distance between the bottom wall and the septum was $102 \mathrm{~mm}$. This probe does not comprise metal except for the elements and battery box. The glass cable which barely affects the electric field in the GTEM cell was used between the probe and control computer. The probe was used to measure the electric field in the $1-16 \mathrm{GHz}$ range. The change of the electric field was defined as the Eq. (1). In order to evaluate the difference in the field strength exactly caused by the insertion of the block as defined by (1), we keep the probe and cable at the same position throughout the measurement.

\subsection{Comparison result}

The effect on the electric field strength was compared with and without the block. We then compare the results obtained from the simulation and measurement of the effect on the electric field. Fig. 2 (b) shows a comparison of the simulation and measurement results for the effect on the electric field. In the simulation, we modeled only the tip of the GTEM cell when investigating the effect of the block on the electric field. The results showed that the electric field strength increased when the block was present. A good agreement was also found between the simulation and measurement results. Therefore, the validity of the simulation results was confirmed. However, during the measurements, we found that ripples occurred at some frequencies. These ripples may be caused by the interaction between the probe and the low dielectric block.

\section{Effects of block size and installation location}

As described in section 3, the electric field strength increased when the block was present at the tip of the GTEM cell. In this section, we moved the electric field probe to a position where is used to evaluate emissions and perform immunity testing. Then, the effect on the electric field was investigated by varying the block sizes. We also investigated the effect on the electric field when the position of the block is arranged far from the coaxial connector.

\subsection{Effect of various block sizes on the electric field}

This section discusses the use of various block sizes and examines the effect on the electric field at a position used to evaluate emissions and perform immunity testing. Fig. 3 (a) shows the outline of the measurement system 


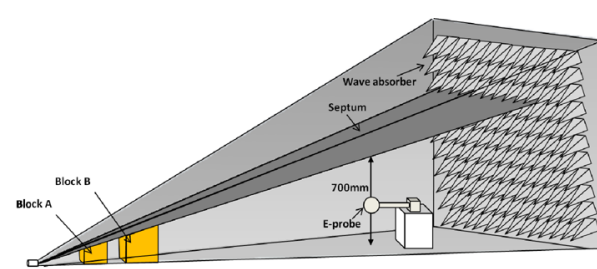

(a) Outline of measurement model for varying block sizes

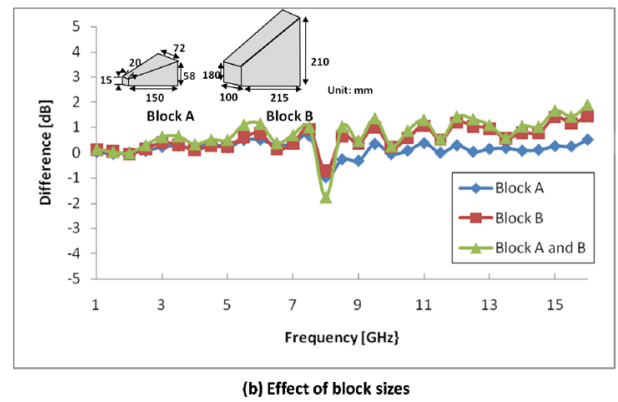

Fig. 3. Measurement system and comparison result.

with blocks of different sizes placed at the tip of the GTEM cell. The electric field probe was set at a point centered between the bottom wall and the septum, which were $700 \mathrm{~mm}$ apart at this location. The probe was then used to measure the electric field. Fig. 3(b) shows an outline of the blocks (Block A and Block B) and the results with regard to the effect of various block sizes on the electric field. We confirmed that the electric field strength increased, as described in section 3, even though the measurement point was far from the block. We then compared the effect of various block sizes on the electric field. The electric field strength increased as the block size increased, especially at high frequency. The maximum difference in the electric field was $2 \mathrm{~dB}$ at $16 \mathrm{GHz}$. We also found that the electric field changed significantly at $8 \mathrm{GHz}$. Even when the block was not installed in the GTEM cell, the electric field still varied greatly at $8 \mathrm{GHz}$ when compared with other frequencies. It is suggested that this change may have been caused by intrinsic characteristics of our GTEM cell.

\subsection{Effect of various block location on the electric field}

As described in sections 3.2 and 4.1, it is possible for the foam block to increase the electric field strength when it is installed at the tip of the GTEM cell. In this section, we investigate the effect on the electric field when the position of the block is arranged far from the tip of the GTEM cell. We varied the installation location of the block to evaluate the effect on the electric field. Fig. 3 (c) shows an outline of the measurement system with different size blocks. These blocks were commercially supplied. The electric field probe was set at the center point, where the distance between the bottom wall and the septum was $700 \mathrm{~mm}$, and was used to measure the electric field. Fig. 3(d) shows an outline of the blocks (Block C and Block D) and the results for the effect on the electric field of various installation locations for the block at the frequency 1,6 and $11 \mathrm{GHz}$. As a result, it was confirmed that variations in size and distance had little influence on the electric field when
(C) IEICE 2009

DOI: $10.1587 /$ elex.6.1608 Received October 09, 2009 Accepted October 20, 2009 Published November 25, 2009 
the block is arranged at a position where is far from the tip of the GTEM cell. This is because, when the block is far from the coaxial connector, the multiple reflections between the coaxial connector and the tip of the block become weaker.

\section{Conclusions}

In this paper, the effect of a low dielectric block placed at the tip of a GTEM cell on the electric field was numerically and experimentally investigated in the $1-16 \mathrm{GHz}$ frequency range. The low dielectric block was found to increase the electric field when it was placed at the tip of the GTEM cell. With only a small change in the electric permittivity, the electric field strength increased greatly. When the size of the low dielectric block was increased, the electric field strength also increased. Therefore, it is possible to use the dielectric block to increase the electric field strength by placing it at the tip of a GTEM cell, especially at high frequency. However, we also confirmed that when the low dielectric block was installed in a location far from the coaxial connector, it had little influence on the electric field, regardless of its size. Therefore, it is possible to use a low dielectric block as a mounting stage which is used for installing the equipment under test to maintain the electric field distribution at high frequency in a GTEM cell.

\section{Acknowledgments}

This work was financially supported from the Ministry of Internal Affairs and Communications (MIC) of the Japanese Government. 